\title{
The effect of rapamycin treatment on cerebral ischemia: A systematic review and meta-analysis of animal
}

model studies.

Daniel J Beard ${ }^{1}$, Gina Hadley ${ }^{1,2}$, Neal Thurley ${ }^{3}$, David W Howells ${ }^{4}$, Brad A Sutherland ${ }^{4}$, Alastair M Buchan ${ }^{1,5,6}$

${ }^{1}$ Acute Stroke Programme, Radcliffe Department of Medicine, University of Oxford, Oxford, UK

${ }^{2}$ Nuffield Department of Clinical Neurosciences, University of Oxford, Oxford, UK.

${ }^{3}$ Bodleian Healthcare Libraries, University of Oxford, Oxford UK.

${ }^{4}$ School of Medicine, College of Health and Medicine, University of Tasmania, Hobart, Australia

${ }^{5}$ Medical Sciences Division, University of Oxford, Oxford, UK

${ }^{6}$ Acute Vascular Imaging Centre, University of Oxford, Oxford University Hospitals, Oxford, UK

Corresponding author:

Professor Alastair Buchan

Medical Sciences Division

University of Oxford

John Radcliffe Hospital

Headington

Oxford

OX3 9DU

tel: +44 (0)1865 220346

email: alastair.buchan@medsci.ox.ac.uk

Key words: experimental stroke; infarct volume; mTOR; rapamycin; systematic review and meta-analysis.

Word count: $\underline{5339}$

\section{List of figures and tables}

Figure 1. Efficacy of rapamycin treatment on infarct volume and behavioral scores.

Figure 2. Meta-regression of the effect of rapamycin dose on outcome

Figure 3. Quality score and publication bias

Table 1. Design characteristics of included studies 


\begin{abstract}
Background: Amplifying endogenous neuroprotective mechanisms is a promising avenue for stroke therapy. One target is mammalian target of rapamycin (mTOR), a serine/threonine kinase regulating cell proliferation, cell survival, protein synthesis and autophagy. Animal studies investigating the effect rapamycin on mTOR inhibition following cerebral ischemia have shown conflicting results.

Aim: To conduct a systematic review and meta-analysis evaluating the effectiveness of rapamycin in reducing infarct volume in animal models of ischemic stroke.

Summary of review: Our search identified 328 publications. Seventeen publications met inclusion criteria (52 comparisons: 30 reported infarct size, 22 reported neurobehavioral score). Study quality was modest (median 4 out of 9) with no evidence of publication bias. The point estimate for the effect of rapamycin was a $21.6 \%$ (95\% CI, $7.6 \%-35.7 \% p<0.01)$ improvement in infarct volume and 30.5\% (95\% CI 17.2\% - 43.8\%, $p<0.0001)$ improvement in neuroscores. Effect sizes were greatest in studies using lower doses of rapamycin.

Conclusion: Low dose rapamycin treatment may be an effective therapeutic option for stroke. Modest study quality means there is a potential risk of bias. We recommend further high quality preclinical studies on rapamycin in stroke before progressing to clinical trials.
\end{abstract}




\section{Introduction}

Amplifying the brain's intrinsic neuroprotective pathways is a promising avenue for developing new treatments for stroke. The serine/threonine kinase Mammalian Target of Rapamycin Complex 1 (mTORC1) plays a role in neuronal degeneration following global ischemia. ${ }^{1}$ If cellular energy is abundant, mTORC1 will be activated leading to protein synthesis, cell proliferation and cytoskeletal formation. ${ }^{2}$ When energy supplies are depleted, mTORC1 is inhibited through the action of the tuberous sclerosis complex (TSC) made up of hamartin (TSC1) and tuberin (TSC2). ${ }^{3,} 4$ Following global ischemia, resistant cells within the cornu ammonus 3 (CA3) subfield of the hippocampus are able to upregulate the expression of hamartin leading to mTORC1 inhibition, limiting protein synthesis and increasing productive autophagy to preserve energy stores and promote cell survival. ${ }^{1}$

Pharmacological inhibition of mTORC1 can be achieved using rapamycin (sirolimus). Rapamycin is an FDA approved immunosuppressant/antirejection treatment for kidney transplantation ${ }^{5}$ and in drug eluting coronary stents preventing intimal hyperplasia and occlusion. ${ }^{6}$ Post-hoc analysis of preclinical data justifying previous candidate neuroprotectants, NXY-059 and Erythropoietin identified significant experimental deficits. ${ }^{7} 8$ Our systematic review and meta-analysis aims to measure the overall efficacy of rapamycin in experimental stroke. This will serve to guide further preclinical studies or if significant neuroprotection is detected with no effects of bias, rapamycin may be rapidly re-purposed as a stroke treatment.

\section{Methods}

Methods were pre-specified in a study protocol (http://www.dcn.ed.ac.uk/camarades/research.html\#protocols) and followed the Systematic Review Protocol for Animal Intervention Studies. ${ }^{9}$

\section{Search Strategy}

The Ovid interface was used to search MEDLINE (1946-May 2017) and EMBASE (1974-May 2017) with no language limits.

The search focused around two keywords "ischemic stroke” and "rapamycin” with respective thesaurus terms and free-text terms. Full search strategies are in supplementary material. 


\section{Inclusion and Exclusion Criteria}

Included studies reported the effects of rapamycin using in vivo animal model of cerebral ischemia with the primary outcome of infarct volume by any method. Secondary outcome measures included neurobehavioral scores, cerebral blood flow (CBF) and blood brain barrier (BBB) permeability. Experiments involving co-treatments with additional drugs or using rapamycin to rescue a genetic knock-down were excluded.

\section{Quality Assessment}

We assessed risk of bias using a 9-item quality checklist (adapted from Collaborative Approach to Meta-Analysis and Review of Animal Data from Experimental Studies (CAMARADES) ${ }^{10}$.

\section{Data Extraction}

Titles and abstracts were screened independently by 2 reviewers (D.J.B and G.H). Full texts were obtained for eligible studies and screened independently (D.J.B and G.H). A third reviewer (B.A.S) arbitrated any differences of opinion. Data were extracted by G.H and D.J.B for study design elements including: species, strain, age, sex of animals used; co-morbidities; anaesthetic, ischemia duration, model of ischemia; dose, route, timing of drug administration, time of outcome assessment; methods of outcome assessment and type of outcome measurement (infarct volume, behaviour, CBF, BBB permeability). For extraction of dose for each comparison, a standardised dose in $\mathrm{mg} / \mathrm{kg}$ was calculated based on the dose of rapamycin administered and the number of times that dose was administered.

For each comparison, data describing the number of animals per group, the mean outcome, and the standard deviation for both the control and treatment group were extracted to enable calculation of a normalised mean difference (NMD). Where studies compared multiple doses with a single control group, individual comparisons were made between each specific dose and the control, with control n numbers divided by the number of doses. Where data were reported graphically, digital ruler software ascertained values, and where data for a single animal group are reported at different time points, data were extracted for the final time point only. 


\section{Meta-analysis}

All meta-analyses and meta-regression were conducted using Stata/SE 15.0 (StataCorp LLC, College Station, TX, USA). Means and standard deviations for infarct volume and behavioral outcomes from each study were used for all meta-analyses. A $p<0.05$ was considered statistically significant. To determine the overall effect of rapamycin treatment on each outcome measure, a meta-analysis was performed using the metan function. For the effect of rapamycin on both infarct volume and neurological deficit behavioural scores in each individual study, NMD was calculated using Equation (1):

$N M D_{i}=100 \% \cdot \frac{\bar{X}_{R x}-\bar{X}_{C}}{\bar{X}_{C}}$

A pooled effect size was determined using the random effects model of DerSimoninan and Laird (estimated heterogeneity taken from the Mantel-Haenszel model). A_heterogeneity of $\mathrm{I}^{2}>50 \%$ indicated significant heterogeneity. To assess correlation between infarct volume and behavioural outcome for studies including both outcomes, a linear regression was performed. To identify which variables contributed to any heterogeneity observed in the infarct volume meta-analysis, meta-regression was conducted using the metareg function. For each independent variable, an adjusted $\mathrm{R}^{2}$ value was calculated determining the proportion of between-study variance explained by the variable. A trim and fill analysis was performed using the metatrim function assessing publication bias.

\section{Results}

A total of 528 records were retrieved (Ovid Medline, 243; Ovid EMBASE, 285), following removal of duplicates, 328 records remained. A further 278 articles were excluded on title and abstract, leaving 64 full text articles. 42 articles were excluded after reading the full text. The remaining 22 full text articles used rapamycin in the treatment of brain ischemia (17 focal cerebral ischemia, 3 neonatal hypoxia-ischemia and 2 global ischemia) (Supplementary Figure 1). Our pre-defined study design stipulated that quantitative assessment would only be carried out on $>10$ observations leaving only focal cerebral ischemia studies. These 17 publications, ${ }^{11-26}$ contained 52 comparisons: 30 comparisons reported data on infarct size and 22 comparisons reported data neurobehavioral score. All studies investigating the effect of rapamycin in either neonatal hypoxia ischemia or global ischemia showed that rapamycin reduced brain damage ${ }^{27-31}$ (Supplementary Tables 1 and 2). 


\section{Efficacy}

The point estimate for the effect of rapamycin was a 21.6\% (95\% CI 7.6-35.7\%, $p<0.05)$ improvement in infarct volume (Figure 1A) and 30.5\% (95\% CI 17.2-43.8\%, $p<0.0001)$ improvement in neuroscores (Figure 1B). There was significant statistical heterogeneity within comparisons of infarct volume $\left(\mathrm{I}^{2}=92 \%\right)$ and neurobehavioral scores $\left(\mathrm{I}^{2}=97.8 \%\right) .22$ of the 30 comparisons of infarct volume also had associated neurobehavioral assessment, enabling a correlation analysis between the two outcome measures. Infarct volume and neurobehavioral scores were highly correlated $\left(\mathrm{R}^{2}=0.7069, p<0.0001\right.$, Figure $\left.1 \mathrm{C}\right)$, showing consistency of improvement by rapamycin as determined by these two outcome measures. In line with our pre-defined selection criteria, we did not perform meta-analysis on other secondary outcome measures of $\mathrm{BBB}$ breakdown and $\mathrm{CBF}$, as there were $<10$ comparisons for each variable. However, 5 out of 6 studies reporting BBB breakdown showed that rapamycin treatment reduced BBB permeability. ${ }^{15,16,21,22,26,32}$ A single study reporting CBF showed that rapamycin non-significantly improved CBF following reperfusion ${ }^{14}$ (Supplementary Table 3).

\section{Effect of study characteristics on efficacy}

Study design characteristics are in Table 1. Only the dose of rapamycin explained a significant amount of betweenstudy heterogeneity $\left(\mathrm{R}^{2}=47 \%, p<0.001\right)$. Lower doses of rapamycin resulted in a greater effect size (Figure $2 \mathrm{~A}$ ). The range of doses used was substantial $(0.000001-40 \mathrm{mg} / \mathrm{kg})$ and so visualization of this correlation was difficult due to the cluster of studies using a $0-1 \mathrm{mg} / \mathrm{kg}$ dose of rapamycin. The dose was log transformed and there is a clear association, with lower doses of rapamycin providing greater protection against injury following cerebral ischemia (Figure 2B). Important to note the two studies using the highest dose of rapamycin $(40 \mathrm{mg} / \mathrm{kg})$ also had the worst outcomes. ${ }^{14,15}$ Other design characteristics such as species, route of administration, dose timing, ischemia model, permanent vs. transient ischemia, ischemia duration, outcome assessment time, comorbidities, randomization, blinded ischemia induction or blinded outcome assessment did not account for a significant portion of between study heterogeneity (Supplementary Table 4 contains all $\mathrm{R}^{2}$ and $p$ values).

Study quality and publication bias 
The median number of study quality checklist items scored was 4 out of a possible 9 (range 3-7) (Supplementary Table 5). Classifying studies by quality score did not account for a significant portion of study heterogeneity $\left(\mathrm{R}^{2}=\right.$ 2.94\%, $p>0.05$ (Figure 3A). The funnel plot with trim and fill analysis did not suggest publication bias (Figure 3B).

\section{Discussion}

This systematic review and meta-analysis supports the overall efficacy of rapamycin improving both histological and functional outcomes in animal models of acute ischemic stroke. Infarct volumes and behavioral scores were highly correlated. There was no evidence of publication bias. The highest levels of efficacy were at lower doses of rapamycin. No other variables had a significant bearing on the efficacy of rapamycin. These findings suggest that low dose rapamycin has the potential to be utilized as a neuroprotective strategy in patients with ischemic stroke.

A global estimate of 30 comparisons revealed that rapamycin significantly reduced infarct volume by $22 \%$. This is slightly lower than a 31\% reduction of infarct volume reported in a systematic review and meta-analysis (with median quality score of 4 and publication bias) of FK506 (Tacrolimus), another immunosuppressive compound. ${ }^{33}$ Unlike rapamycin, FK506 binding to FKBP12 does not induce mTOR inhibition and changes in autophagy. ${ }^{34}$ Rather, the FK506-FKBP12 complex blocks the activation of calcineurin and downstream signaling pathways, resulting in neuroprotection. ${ }^{35}$ These different mechanisms of action may explain the difference in efficacy between the two treatments. FK506 has been trialed in human stroke but failed due to adverse side effects. ${ }^{36}$ Though our dataset does not indicate an influence of confounding factors such as randomization or blinding on rapamycin effect size, there may still be a high risk of bias given the smaller number of studies and moderate study quality.

The dose of rapamycin accounted for significant variability in the model. Specifically, lower doses of rapamycin showed greater efficacy at reducing infarct volume than higher doses. Doses used in this study ranged from $<1 \mathrm{mg} / \mathrm{kg}$ to $40 \mathrm{mg} / \mathrm{kg}$. If one takes into account allometric scaling between rats and humans, a $40 \mathrm{mg} / \mathrm{kg}$ dose in a $300 \mathrm{~g}$ rat would equate to $10 \mathrm{mg} / \mathrm{kg}$ in a $70 \mathrm{~kg}$ human. ${ }^{37}$ This dose is 50 times higher than that used in humans for kidney transplantation $(0.2 \mathrm{mg} / \mathrm{kg} /$ day $){ }^{38}$ This is in contrast with FK506, in which animal studies used doses that more closely resembled doses used clinically. ${ }^{33}$ FK506 efficacy showed a slight trend towards an inverted U shaped dose

response curve. ${ }^{33}$ Studies using higher doses of rapamycin were published in 2016. The authors do not to give a 
rationale for using such high doses of rapamycin. Such an increase in the dose used may be as a result of a drop in the price of rapamycin following the patent (US5100899A) expiring in 2013.

Though our dose findings seem counterintuitive, there are two sets of observations that suggest they make sense from a biological perspective. Inhibition of mTORC1 increases autophagy.$^{39}$ Basal levels of autophagy are necessary for neuronal health (neurodegeneration and ubiquitinated protein aggregates occur in animal models lacking this process). ${ }^{40}$ At low doses, rapamycin may be beneficial but harmful at high doses because of unregulated autophagy. A second possible explanation is that prolonged rapamycin treatment can affect the assembly and function of mTORC2. ${ }^{41}$ mTORC2 plays an important role in cell survival during stress. ${ }^{42}$ Studies utilizing low doses of rapamycin may also have stimulated autophagy enough without inhibiting mTORC2 to enhance neuronal survival following ischemia. Higher dose studies may have over-stimulated autophagy and/or inhibited mTORC2 leading to increased infarct volume. ${ }^{14}$ A number of studies have shown that adverse effects such as immunosuppression and glucose intolerance ${ }^{43}$ are mediated by mTORC2 inhibition due to prolonged rapamycin treatment. ${ }^{44}$ Experimental evidence from rodents suggests that a one off bolus of $1 \mathrm{mg} / \mathrm{kg}$ of rapamycin reduces peripheral mTORC1 activity for 1-3 days without suppressing mTORC2 and ultimately improves immunological function and reduces glucose intolerance in these animals. ${ }^{43}$ Use of rapamycin as an acute therapy inhibiting mTORC1 in brain without peripheral suppression of mTORC2 may improve its side effect profile. Future preclinical trials of rapamycin in stroke and potential safety trials in humans should assess mTORC1 and mTORC2 activity levels in peripheral immune cells, overall immune cell numbers and glucose tolerance tests. ${ }^{43}$

Efficacy of rapamycin was not affected by timing of drug administration though many of the studies provided therapy before or early after onset of stroke (Table 1) and equally efficacious in permanent and temporary ischemia models. Rapamycin could be a stroke treatment not bound by time or imaging constraints, used for patients who are outside the time window for thrombolysis/thrombectomy or as an adjunctive therapy to this gold standard treatment. Inhibiting mTORC1 affects numerous cell death, CBF, BBB and inflammatory pathways that develop within minutes to days after stroke onset. ${ }^{17,45-47}$ Patients with transient ischemic attacks (TIAs), who have an increased 90 day stroke risk may also benefit. ${ }^{48,}{ }^{49}$ In 313 Mayo Clinic heart transplant patients on Sirolimus (rapamycin) followed over 20 years, not a single patient in this inherently high-risk group had a stroke or TIA. ${ }^{50} \underline{I n}$ a similar sized study at the same 
institution, $5 \%$ of patients not taking sirolimus developed cerebrovascular events. ${ }^{51}$ Rapamycin may therefore have applications in the prevention and treatment of ischemic stroke.

Further high quality pre-clinical studies of rapamycin in stroke are needed before progressing to clinical trial. No studies in this review provided a sample size calculation. Only 8 of 17 studies conducted blinded outcome assessment with a trend toward increased effect size in blinded studies. The only co-morbidity reported in this review was hypertension (3 studies) with a trend towards lower efficacy in hypertensive animals. Rigorous multi-centre preclinical trials have been used previously to assess candidate preclinical drugs before progression to clinical trial (Interleukin-1 Receptor Antagonist and anti-CD49d antibodies). ${ }^{52,53}$ The design of such a trial of rapamycin, learning from the shortcomings of previous preclinical studies of rapamycin, will improve the overall study quality, improve our confidence in taking this therapy forward to clinical trials and ultimately increase the translational impact of preclinical rapamycin stroke research. ${ }^{54}$

Systematic reviews and meta-analyses have limitations. Negative studies are less likely to be published overestimating efficacy. Previous meta-analyses have shown that average neuroprotective efficacy is $\sim 31 \%$, which is often adjusted to an actual estimate of efficacy of around $20 \%$, accounting for publication bias. ${ }^{55}$ Interestingly, the effect size of rapamycin was a $21 \%$ reduction in infarct volume, with trim and fill analysis suggesting no evidence of publication bias in studies identified. It must be noted that the small number of studies in this analysis means that there may be limited power to detect subgroup effects and publication bias. ${ }^{56}$ However, the lack of publication bias seems in line with the general observation that 4 comparisons showed that rapamycin was not neuroprotective, making infarct volume worse, yet these studies were still published. Our analysis has revealed that 3 of these 4 studies utilized supra-therapeutic doses of rapamycin (5-40mg/kg), the most likely explanation for exacerbated injury seen in these studies.

In conclusion rapamycin is neuroprotective in animal models of ischemic stroke. Reduced infarct volumes were highly correlated with improvements in neurological outcome. Clinically relevant lower doses of rapamycin showed the greatest efficacy in reducing infarct volume. The modest quality of the studies and large heterogeneity between 
studies means that further high quality (multicenter randomized) preclinical trials are required to determine the suitability of rapamycin as a treatment for human stroke.

\section{Acknowledgements}

AMB, BAS and DJB were funded by the Medical Research Council UK (MR/M022757/1). BAS was funded by the National Health and Medical Research Council (APP1137776), Rebecca L. Cooper Foundation, J J Mason and H S Williams Memorial Foundation, Brain Foundation and Royal Hobart Hospital Research Foundation. GH was supported by Oxford University Clinical Academic Graduate School, Oxford

\section{Conflicts of Interest}

AMB is a senior medical science advisor and co-founder of Brainomix, a company that develops electronic ASPECTS (e-ASPECTS), an automated method to evaluate ASPECTS in stroke patients. All other authors declare no conflict of interest.

\section{$\underline{\text { References }}$}

1. Papadakis M, Hadley G, Xilouri M, et al. Tsc1 (hamartin) confers neuroprotection against ischemia by inducing autophagy. Nat Med 2013; 19: 351-357. DOI: 10.1038/nm.3097.

2. Wang C, Yu JT, Miao D, et al. Targeting the mTOR signaling network for Alzheimer's disease therapy. Mol Neurobiol 2014; 49: 120-135. DOI: 10.1007/s12035-013-8505-8.

3. DeYoung MP, Horak P, Sofer A, et al. Hypoxia regulates TSC1/2-mTOR signaling and tumor suppression through REDD1-mediated 14-3-3 shuttling. Genes Dev 2008; 22: 239-251. DOI: 10.1101/gad.1617608.

4. Hardie DG. The AMP-activated protein kinase pathway--new players upstream and downstream. J Cell Sci 2004; 117: 5479-5487. DOI: 10.1242/jcs.01540.

5. Saunders RN, Metcalfe MS and Nicholson ML. Rapamycin in transplantation: a review of the evidence. Kidney Int 2001; 59: 3-16. DOI: 10.1046/j.1523-1755.2001.00460.x.

6. McKeage K, Murdoch D and Goa KL. The sirolimus-eluting stent: a review of its use in the treatment of coronary artery disease. Am J Cardiovasc Drugs 2003; 3: 211-230.

7. Jerndal M, Forsberg K, Sena ES, et al. A systematic review and meta-analysis of erythropoietin in experimental stroke. J Cereb Blood Flow Metab 2010; 30: 961-968. DOI: 10.1038/jcbfm.2009.267.

8. Macleod MR, van der Worp HB, Sena ES, et al. Evidence for the efficacy of NXY-059 in experimental focal cerebral ischaemia is confounded by study quality. Stroke 2008; 39: 2824-2829. DOI: 10.1161/STROKEAHA.108.515957.

9. $\quad$ de Vries RBMH, C.R.; Langendam, M.W.; van Luijk, J.; Leenaars, M.; Merel Ritskes-Hoitinga, M.; Wever, K.W. A protocol format for the preparation, registration and publication of systematic reviews of animal intervention studies. Evidence-based Preclinical Medicine 2015; 1: 1-9.

10. Macleod MR, O'Collins T, Howells DW, et al. Pooling of animal experimental data reveals influence of study design and publication bias. Stroke 2004; 35: 1203-1208. DOI: 10.1161/01.STR.0000125719.25853.20. 
11. Bochelen D, Rudin M and Sauter A. Calcineurin inhibitors FK506 and SDZ ASM 981 alleviate the outcome of focal cerebral ischemic/reperfusion injury. J Pharmacol Exp Ther 1999; 288: 653-659.

12. Buckley KM, Hess DL, Sazonova IY, et al. Rapamycin up-regulation of autophagy reduces infarct size and improves outcomes in both permanent MCAL, and embolic MCAO, murine models of stroke. Exp Transl Stroke Med 2014; 6: 8. DOI: 10.1186/2040-7378-6-8.

13. Chauhan A, Sharma U, Jagannathan NR, et al. Rapamycin protects against middle cerebral artery occlusion induced focal cerebral ischemia in rats. Behav Brain Res 2011; 225: 603-609. DOI: 10.1016/j.bbr.2011.08.035.

14. Chi OZ, Barsoum S, Vega-Cotto NM, et al. Effects of rapamycin on cerebral oxygen supply and consumption during reperfusion after cerebral ischemia. Neuroscience 2016; 316: 321-327. DOI: 10.1016/j.neuroscience.2015.12.045.

15. Chi OZ, Mellender SJ, Barsoum S, et al. Effects of rapamycin pretreatment on blood-brain barrier disruption in cerebral ischemia-reperfusion. Neuroscience letters 2016; 620: 132-136. DOI: 10.1016/j.neulet.2016.03.053.

16. Guo W, Feng GY, Miao YY, et al. Rapamycin alleviates brain edema after focal cerebral ischemia reperfusion in rats. Immunopharm Immunot 2014; 36: 211-223. DOI: 10.3109/08923973.2014.913616.

17. Li D, Wang C, Yao Y, et al. mTORC1 pathway disruption ameliorates brain inflammation following stroke via a shift in microglia phenotype from M1 type to M2 type. FASEB J 2016; 30: 33883399. DOI: 10.1096/fj.201600495R.

18. Li Q, Zhang T, Wang JX, et al. Rapamycin attenuates mitochondrial dysfunction via activation of mitophagy in experimental ischemic stroke. Biochem Bioph Res Co 2014; 444: 182-188. DOI: 10.1016/j.bbrc.2014.01.032.

19. Qi HY, Su FY, Wan S, et al. The Antiaging Activity and Cerebral Protection of Rapamycin at Micro-doses. Cns Neurosci Ther 2014; 20: 991-998. DOI: 10.1111/cns.12338.

20. Sharkey J and Butcher SP. Immunophilins Mediate the Neuroprotective Effects of Fk506 in Focal Cerebral-Ischemia. Nature 1994; 371: 336-339. DOI: DOI 10.1038/371336a0.

21. Sheng R, Zhang LS, Han R, et al. Autophagy activation is associated with neuroprotection in a rat model of focal cerebral ischemic preconditioning. Autophagy 2010; 6: 482-494. DOI: DOI 10.4161/auto.6.4.11737.

22. Su JY, Zhang TT, Wang KW, et al. Autophagy Activation Contributes to the Neuroprotection of Remote Ischemic Perconditioning Against Focal Cerebral Ischemia in Rats. Neurochemical Research 2014; 39: 2068-2077. DOI: 10.1007/s11064-014-1396-x.

23. Xie L, Sun F, Wang J, et al. mTOR signaling inhibition modulates macrophage/microgliamediated neuroinflammation and secondary injury via regulatory T cells after focal ischemia. $J$ Immunol 2014; 192: 6009-6019. DOI: 10.4049/jimmunol.1303492.

24. Xie R, Wang P, Cheng M, et al. Mammalian Target of Rapamycin Cell Signaling Pathway Contributes to the Protective Effects of Ischemic Postconditioning Against Stroke. Stroke 2014; 45: 2769+. DOI: 10.1161/Strokeaha.114.005406.

25. Yan WJ, Zhang HP, Bai XG, et al. Autophagy activation is involved in neuroprotection induced by hyperbaric oxygen preconditioning against focal cerebral ischemia in rats. Brain research 2011; 1402: 109-121. DOI: 10.1016/j.brainres.2011.05.049.

26. Yang Z, Zhong LN, Zhong SC, et al. Hypoxia induces microglia autophagy and neural inflammation injury in focal cerebral ischemia model. Exp Mol Pathol 2015; 98: 219-224. DOI: 10.1016/j.yexmp.2015.02.003.

27. Carloni S, Albertini MC, Galluzzi L, et al. Increased autophagy reduces endoplasmic reticulum stress after neonatal hypoxia-ischemia: role of protein synthesis and autophagic pathways. Exp Neurol 2014; 255: 103-112. DOI: 10.1016/j.expneurol.2014.03.002.

28. Carloni S, Buonocore G and Balduini W. Protective role of autophagy in neonatal hypoxiaischemia induced brain injury. Neurobiol Dis 2008; 32: 329-339. DOI: 10.1016/j.nbd.2008.07.022. 
29. Carloni S, Girelli S, Scopa C, et al. Activation of autophagy and Akt/CREB signaling play an equivalent role in the neuroprotective effect of rapamycin in neonatal hypoxia-ischemia. Autophagy 2010; 6: 366-377.

30. Dong F, Yao R, Yu H, et al. Neuroprotection of Ro25-6981 Against Ischemia/ReperfusionInduced Brain Injury via Inhibition of Autophagy. Cell Mol Neurobiol 2017; 37: 743-752. DOI: 10.1007/s10571-016-0409-5.

31. Yang X, Hei C, Liu P, et al. Inhibition of mTOR Pathway by Rapamycin Reduces Brain Damage in Rats Subjected to Transient Forebrain Ischemia. Int J Biol Sci 2015; 11: 1424-1435. DOI: 10.7150/ijbs.12930.

32. Yin LL, Ye SS, Chen Z, et al. Rapamycin Preconditioning Attenuates Transient Focal Cerebral Ischemia/Reperfusion Injury in Mice. Int $J$ Neurosci 2012; 122: 748-756. DOI: 10.3109/00207454.2012.721827.

33. Macleod MR, O'Collins T, Horky LL, et al. Systematic review and metaanalysis of the efficacy of FK506 in experimental stroke. J Cerebr Blood F Met 2005; 25: 713-721. DOI: 10.1038/sj.jcbfm.9600064.

34. Cardenas ME, Sanfridson A, Cutler NS, et al. Signal-transduction cascades as targets for therapeutic intervention by natural products. Trends Biotechnol 1998; 16: 427-433. DOI: Doi 10.1016/S0167-7799(98)01239-6.

35. Li JY, Furuichi Y, Matsuoka N, et al. Tacrolimus (FK506) attenuates biphasic cytochrome c release and Bad phosphorylation following transient cerebral ischemia in mice. Neuroscience 2006; 142: 789-797. DOI: 10.1016/j.neuroscience.2006.06.064.

36. Ishii $\mathrm{T}$, Asai $\mathrm{T}$, Oyama $\mathrm{D}$, et al. Treatment of cerebral ischemia-reperfusion injury with PEGylated liposomes encapsulating FK506. FASEB J 2013; 27: 1362-1370. DOI: 10.1096/fj.12-221325.

37. West GB and Brown JH. The origin of allometric scaling laws in biology from genomes to ecosystems: towards a quantitative unifying theory of biological structure and organization. $J$ Exp Biol 2005; 208: 1575-1592. DOI: 10.1242/jeb.01589.

38. Knight RJ and Kahan BD. The place of sirolimus in kidney transplantation: Can we reduce calcineurin inhibitor renal toxicity? Kidney International 2006; 70: 994-999. DOI: 10.1038/sj.ki.5001644.

39. Boland B, Kumar A, Lee S, et al. Autophagy induction and autophagosome clearance in neurons: Relationship to autophagic pathology in Alzheimer's disease. Journal of Neuroscience 2008; 28: 69266937. DOI: 10.1523/Jneurosci.0800-08.2008.

40. Cherra SJ, Dagda RK and Chu CT. Review: Autophagy and neurodegeneration: survival at a cost? Neuropathology and applied neurobiology 2010; 36: 125-132. DOI: 10.1111/j.13652990.2009.01062.x.

41. Sarbassov DD, Ali SM, Sengupta S, et al. Prolonged rapamycin treatment inhibits mTORC2 assembly and Akt/PKB. Molecular Cell 2006; 22: 159-168. DOI: 10.1016/j.molcel.2006.03.029.

42. Shin S, Buel GR, Wolgamott L, et al. ERK2 Mediates Metabolic Stress Response to Regulate Cell Fate. Mol Cell 2015; 59: 382-398. DOI: 10.1016/j.molcel.2015.06.020.

43. Arriola Apelo SI, Neuman JC, Baar EL, et al. Alternative rapamycin treatment regimens mitigate the impact of rapamycin on glucose homeostasis and the immune system. Aging Cell 2016; 15: 28-38. DOI: $10.1111 /$ acel.12405.

44. Arriola Apelo SI and Lamming DW. Rapamycin: An InhibiTOR of Aging Emerges From the Soil of Easter Island. J Gerontol A Biol Sci Med Sci 2016; 71: 841-849. DOI: 10.1093/gerona/glw090.

45. Li H, Gao A, Feng D, et al. Evaluation of the protective potential of brain microvascular endothelial cell autophagy on blood-brain barrier integrity during experimental cerebral ischemiareperfusion injury. Transl Stroke Res 2014; 5: 618-626. DOI: 10.1007/s12975-014-0354-x.

46. Lin AL, Zheng W, Halloran JJ, et al. Chronic rapamycin restores brain vascular integrity and function through NO synthase activation and improves memory in symptomatic mice modeling Alzheimer's disease. J Cereb Blood Flow Metab 2013; 33: 1412-1421. DOI: 10.1038/jcbfm.2013.82.

47. Shi ZY, Deng JX, Fu S, et al. Protective effect of autophagy in neural ischemia and hypoxia: Negative regulation of the Wnt/beta-catenin pathway. Int J Mol Med 2017; 40: 1699-1708. DOI: 10.3892/ijmm.2017.3158. 
48. Rothwell PM and Warlow CP. Timing of TIAs preceding stroke - Time window for prevention is very short. Neurology 2005; 64: 817-820. DOI: Doi 10.1212/01.Wnl.0000152985.32732.Ee.

49. Thacker EL, Wiggins KL, Rice KM, et al. Short-term and long-term risk of incident ischemic stroke after transient ischemic attack. Stroke 2010; 41: 239-243. DOI: 10.1161/STROKEAHA.109.569707.

50. van de Beek D, Kremers WK, Kushwaha SS, et al. No Major Neurologic Complications With Sirolimus Use in Heart Transplant Recipients. Mayo Clin Proc 2009; 84: 330-332.

51. van de Beek D, Kremers W, Daly RC, et al. Effect of neurologic complications on outcome after heart transplant. Arch Neurol 2008; 65: 226-231. DOI: DOI 10.1001/archneurol.2007.52.

52. Llovera G, Hofmann K, Roth S, et al. Results of a preclinical randomized controlled multicenter trial (pRCT): Anti-CD49d treatment for acute brain ischemia. Science translational medicine 2015; 7: 299ra121. DOI: 10.1126/scitranslmed.aaa9853.

53. Maysami S, Wong R, Pradillo JM, et al. A cross-laboratory preclinical study on the effectiveness of interleukin-1 receptor antagonist in stroke. J Cereb Blood Flow Metab 2016; 36: 596-605. DOI: 10.1177/0271678X15606714.

54. Dirnagl U, Hakim A, Macleod M, et al. A concerted appeal for international cooperation in preclinical stroke research. Stroke 2013; 44: 1754-1760. DOI: 10.1161/STROKEAHA.113.000734.

55. Sena ES, van der Worp HB, Bath PM, et al. Publication bias in reports of animal stroke studies leads to major overstatement of efficacy. PLoS Biol 2010; 8: e1000344. DOI: 10.1371/journal.pbio.1000344.

56. Wang QL, J.; Hair, K.;Bannach-Brown, A.; Bahor, Z.; Currie, G.L.; McCann, S.K.; Howells, D.W.; Sena, E.S.; Macleod, M.R. Estimating the statistical performance of different approaches to metaanalysis of data from animal studies in identifying the impact of aspects of study design. Biorxiv 2018. DOI: https://doi.org/10.1101/256776.

57. Xie LK, Sun F, Wang JX, et al. mTOR Signaling Inhibition Modulates Macrophage/MicrogliaMediated Neuroinflammation and Secondary Injury via Regulatory T Cells after Focal Ischemia. Journal of Immunology 2014; 192: 6009-6019. DOI: 10.4049/jimmunol.1303492.

\section{Figure Legends.}

Figure 1. Efficacy of rapamycin treatment on infarct volume and behavioral scores. Point estimate and 95\% CIs for global estimate of (A) infarct volume for each of 30 comparisons and (B) neurobehavioral score for each of 22 comparisons. Effect size is improvement in treated animals expressed as a proportion of outcome in control animals. The size of the filled square for each study indicates the weighting in the global estimate. Diamond and dashed vertical line indicate the global estimate. Solid vertical line represents where treatment and control are equal. (C) Linear regression of infarct volume versus neurobehavioral score. Broken horizontal line represents where the treatment and control are equal for infarct volume. Broken vertical line represents where the treatment and control are equal for neurobehavioral score.

Figure 2. Meta-regression of the effect of rapamycin dose on outcome. (A) Meta-regression of infarct volume effect size plotted against standardized dose of rapamycin. Dose of rapamycin accounted for a significant portion of the 
heterogeneity of infarct volume effect size. Lower doses of rapamycin having a greater reduction in infarct volume. (B) Due to the clustering of studies using doses of rapamycin between $0-1 \mathrm{mg} / \mathrm{kg}$, infarct volume was plotted against the log standardized dose of rapamycin, where the association is more apparent. Weighting of studies represented by size of the circles.

Figure 3. Quality score and publication bias. (A) Point estimates of infarct volume effect size by reported study quality score (adapted from CAMARADES checklist). Weighting of each study for meta-regression is indicated by the size of the circle. Study quality score did not significantly account for effect size. (B) Filled funnel plot of a trim and fill analysis of point estimates of infarct volume effect size to determine any publication bias. Global estimate of effect size as well as pseudo 95\% confidence limits are presented. There is no evidence of publication bias. 
Tables.

Table 1. Design characteristics of included studies.

\begin{tabular}{|c|c|c|c|c|c|c|c|c|c|c|c|}
\hline Publications & Year & Sex & $\mathbf{N}(\mathrm{C})$ & $\mathbf{N}(\mathbf{R x})$ & $\begin{array}{c}\text { Dose Range } \\
\text { (mg/kg) }\end{array}$ & Species & Time to treatment & Anesthetic & Temp/Perm & Route of Delivery & Outcome \\
\hline Sharkey ${ }^{20}$ & 1994 & Male & $7-13$ & $7-13$ & 1 & Rat & 1 min after & Halothane & Temporary & i.v. & Infarct \\
\hline Bochelen ${ }^{11}$ & 1999 & Male & 18 & 9 & 2 & Rat & 20 min before & Isoflurane & Temporary & i.v. & Infarct \\
\hline Sheng ${ }^{21}$ & 2010 & Male & 6 & 6 & $\begin{array}{l}0.000028- \\
0.000110\end{array}$ & Rat & 24 hour before & Chloral Hydrate & Permanent & i.c.v. & Infarct/Behavior/BBB \\
\hline Chauhan $^{13}$ & 2011 & Male & 8 & 8 & $0.05-0.25$ & Rat & 1 hour before & Chloral Hydrate & Temporary & i.p. & Infarct/Behavior \\
\hline $\operatorname{Yan}^{25}$ & 2011 & Male & 10 & 10 & 0.000001 & Rat & 24 hour before & Pentobarbital Sodium & Temporary & i.c.v. & Infarct/Behavior \\
\hline Yin $^{32}$ & 2012 & Mixed & 4 & 4 & 0.023 & Mouse & 20 min before & Chloral Hydrate & Temporary & i.c & Infarct/Behavior/BBB \\
\hline Buckley $^{12}$ & 2014 & Male & $9-10$ & $7-9$ & 2.5 & Mouse & 0 and 24 hours after & NK & Permanent & i.p. & Infarct/Behavior \\
\hline $\mathrm{Guo}^{16}$ & 2014 & Male & 6 & 6 & 0.25 & Rat & 1 hour after & Pentobarbital Sodium & Temporary & i.p. & Infarct/BBB \\
\hline $\mathrm{Li}^{18}$ & 2014 & Male & 8 & 8 & 0.000028 & Rat & 30 min before & Ketamine Xylazine & Temporary & i.c.v. & Infarct/Behavior \\
\hline $\mathrm{Qi}^{19}$ & 2014 & Male & 10 & 10 & $0.0003-3$ & Mouse/Rat & 3 days before & Isoflurane & Both & i.p. & Infarct/Behavior \\
\hline $\mathrm{Su}^{22}$ & 2014 & Male & 6 & 6 & 0.0001 & Rat & Before (not specified) & Chloral Hydrate & Temporary & i.c.v. & Infarct/Behavior/BBB \\
\hline $\mathrm{Xie}^{57}$ & 2014 & Male & 7 & 7 & 0.001 & Rat & 6 hours after & Isoflurane & Temporary & i.c.v. & Infarct/Behavior \\
\hline $\mathrm{Xie}^{24}$ & 2014 & Male & 8 & 8 & 0.014 & Rat & 1 hour before & Isoflurane & Permanent & i.c.v. & Infarct \\
\hline Yang $^{31}$ & 2015 & Male & 10 & 10 & 4.5 & Mouse & 10 min before & Isoflurane & Permanent & i.c.v. & Infarct/BBB \\
\hline $\mathrm{Chi}^{14}$ & 2016 & Male & 4 & 4 & 40 & Rat & 2 days before & Isoflurane & Temporary & i.p. & Infarct/CBF \\
\hline $\mathrm{Chi}^{15}$ & 2016 & Male & 6 & 6 & 40 & Rat & 2 days before & Isoflurane & Temporary & i.p. & Infarct/BBB \\
\hline $\mathrm{Li}^{17}$ & 2016 & Male & 10 & 10 & 20 & Mouse & 6 hours after & Chloral Hydrate & Temporary & 0 & Infarct/Behavior \\
\hline
\end{tabular}


Number of animals in control group $(\mathrm{n}(\mathrm{C}))$; number of animals in experimental group ( $(\mathrm{Rx})$ ); dose range; species; interval from onset of ischemia to start of treatment; anaesthetic used; permanent or temporary ischemia; route of drug delivery and outcomes measured. N.K, not known; i.v., intravenous; i.c., intracerebral; i.c.v., intracerebroventricular; i.p., intraperitoneal; o., oral, Infarct, infarct volume measurement; behaviour, neurobehavioral outcome; BBB, blood brain barrier permeability, CBF, cerberal blood flow 
